\title{
HYPERGEOMETRIC OPERATOR SERIES AND RELATED PARTIAL DIFFERENTIAL EQUATIONS
}

\author{
BY \\ L. R. BRAGG $\left({ }^{1}\right)$
}

1. Introduction. In several earlier papers ([3]-[7]), J. W. Dettman and the author introduced and made application of the notion of related partial differential equations. Two initial-boundary value problems are said to be related if a solution of one of them is obtained by performing a transformation on a solution of the other. An elementary calculus for treating a number of such related problems was developed in [6]. In some cases, these connections permitted the proving of uniqueness [3]. The purpose of this paper is to extend this calculus to a broader class of problems through the introduction and use of hypergeometric operator series and their properties.

In order to motivate the ideas of this paper, we introduce some formalism. Let

$$
\sum_{j=0}^{k} f_{j}(\tau) y^{(k-j)}(\tau)=0
$$

be a linear ordinary differential equation with polynomial coefficients, having an ordinary or regular singular point at, say, $\tau=0$. Assume further that this equation has a series solution $\sum_{j=0}^{\infty} a_{j} \tau^{j}$ whose coefficients are connected by a two term recurrence formula. Then, such a series can be expressed in terms of a hypergeometric series. If we introduce the change of scale $\tau=\lambda t$, the above equation becomes

$$
\sum_{j=0}^{k} \lambda^{j} f_{j}(\lambda t) D_{t}^{k-j} y(t \lambda)=0
$$

Taking $\lambda=P(x, D)$, a linear partial differential operator independent of $t$ and $D_{t}$, the equation obtained is an "operator differential equation" with the formal operator solution $\sum_{j=0}^{\infty} a_{j} P^{j}(x, D)$. If $\phi(x)$ is a function having suitable analyticity properties, the function

$$
u(x, t)=\sum_{j=0}^{\infty} a_{j} t^{j} P^{j}(x, D) \phi(x)
$$

is then a formal solution of the partial differential equation

$$
\sum_{j=0}^{k} f_{j}(t P(x, D)) P^{j}(x, D) \cdot D_{t}^{k-j} u(x, t)=0 .
$$

If $a_{0}=1$, then $u(x, 0)=\phi(x)$.

Received by the editors July 26, 1968 .

( $\left.{ }^{1}\right)$ This research was supported by NSF Grant GP-7382. 
The difficulty with this formalism is that it assumes that a meaning can be attached to the series $\sum_{j=0}^{\infty} a_{j} t^{j} P^{j}(x, D) \phi(x)$. This requires that $\phi(x)$ be, at least, analytic. The precise requirement depends upon the operator $P(x, D)$ and the coefficients $a_{j}$. In some cases, this formal series can be identified with a solution operator that requires less restrictive assumptions on the function $\phi(x)$. For example, if $a_{j}=(j !)^{-1}$ and $P(x, D)=D_{x}$, then this series can be interpreted as the translation $\phi(x+t)$ if $\phi(x) \in C^{1}$. Similarly, if $a_{j}=(j !)^{-1}$ and $P(x, D)=D_{x}^{2}$, this series can be identified with the Poisson integral formula which defines the solution of the standard heat equation. The concern here will be with identifying other such series, with less restrictive solution operators, through related partial differential equations.

For this purpose, let $O_{1}(t P(x, D))$ denote the above solution operator series for a problem $\pi_{1}$. Assume that $O_{1}(t P(x, D))$ can be expressed in terms of a solution operator series $O_{2}(t P(x, D))=\sum_{j=0}^{\infty} b_{j} t^{j} P^{j}(x, D)$ for a simpler and solvable problem $\pi_{2}$. Then, we can use this relationship to motivate a connection between the solution functions of the pair of problems $\pi_{1}$ and $\pi_{2}$ attached to these two operator series. The correctness of the resulting relationship between the solutions of the two problems can then be proved directly. The continuity requirements on $\phi(x)$ are then dictated by this established relationship between solution functions and the operator $P(x, D)$. The notions of well-posedness and ill-posedness are clearly involved in this. If a fundamental solution is known for $\pi_{2}$, the relationship may permit the construction of a fundamental solution for $\pi_{1}$.

In order to have a mathematical model suitable for proofs, we restrict ourselves to hypergeometric type differential equations and the corresponding hypergeometric operators ${ }_{p} F_{q}\left(\alpha_{1}, \ldots, \alpha_{p} ; \beta_{1}, \ldots, \beta_{q} ; t P\right)$. Numerous other equations can be reduced to this type by appropriate changes of variables. Some of the basic notations, definitions, and properties of the hypergeometric functions suitable for subsequent developments will be discussed in $\S 2$. These properties will be stated as theorems involving shifts on the indices $p$ and $q$, or on the parameters $\alpha_{i}$ or $\beta_{i}$. We also define $P(x, D)$ there with more precision and note the meaning of $e^{t P} \phi(x)$ for certain $P(x, D)$. Those formulas of $\S 2$ that pertain to shifts on the indices $p$ and $q$ will be used in $\$ 3$ to motivate, state, and prove theorems concerning the relationship between solutions of problems of partial differential equations whose underlying equations are possibly of different orders. The proofs will indicate why $P(x, D)$ can, in some real sense, be regarded as a parameter as mentioned earlier. The formulas involving shifts on the parameters $\alpha_{i}$ and $\beta_{i}$ are used in $\S 4$ to reduce problems to a form that permit an application of the results in $\S 3$. Applications of these results will be given in $\$ \S 5$ and 6 . The last of these will be concerned with an interpretation of the theorems of $\S 4$ for the Euler-Poisson-Darboux equation. It will be seen that many of the results obtained by A. Weinstein and his collaborators follow almost immediately. The use of the tools of ordinary differential equations greatly simplifies many considerations. 
Theorems on uniqueness will not be discussed except in special cases. These usually depend upon having more information than is needed for the type of result proved here. When additional information is supplied, uniqueness often readily follows from the relations between the solutions.

2. Generalized hypergeometric functions. In this section, we recall some of the basic definitions and properties of the generalized hypergeometric functions needed for the ensuing development. Additional results pertaining to these functions can be found in [10] and its references.

The generalized hypergeometric function ${ }_{p} F_{q}$ is defined by the basic formula

$$
{ }_{p} F_{q}\left(\alpha_{1}, \ldots, \alpha_{p} ; \beta_{1}, \ldots, \beta_{q} ; z\right)=1+\sum_{n=1}^{\infty} \frac{\prod_{i=1}^{p}\left(\alpha_{i}\right)_{n}}{\prod_{j=1}^{q}\left(\beta_{j}\right)_{n}} \frac{z^{n}}{n !}
$$

where $(\sigma)_{n}=\sigma(\sigma+1) \cdots(\sigma+n-1)$. The function is unchanged by permuting the $\alpha_{i}$ 's among themselves and the $\beta_{j}$ 's among themselves. We use the notation ${ }_{0} F_{q}\left(-; \beta_{1}, \ldots, \beta_{q} ; z\right)$ if $p=0$ and define $\prod_{i=1}^{p}\left(\alpha_{i}\right)_{n}=1$ if $p=0$. A similar definition holds for ${ }_{p} F_{0}\left(\alpha_{1}, \ldots, \alpha_{p} ;-; z\right)$. If some of the $\alpha_{i}$ are nonpositive integers, then (2.1) reduces to a polynomial in $z$. None of the $\beta_{j}$ are permitted to be nonpositive integers in (2.1). If $p \leqq q$, the series (2.1) converges for all finite $z$ and if $p=q+1$, this series converges for $|z|<1$ and diverges for $|z|>1$. The case $p>q+1$ is of no interest since the series (2.1) then only converges at $z=0$.

For certain purposes, it is more convenient to rewrite (2.1) in terms of the gamma function as

$$
{ }_{p} F_{q}=\frac{\prod_{j=1}^{q} \Gamma\left(\beta_{j}\right)}{\prod_{i=1}^{p} \Gamma\left(\alpha_{i}\right)} \sum_{n=0}^{\infty} \frac{\prod_{i=1}^{p} \Gamma\left(\alpha_{i}+n\right)}{\prod_{j=1}^{q} \Gamma\left(\beta_{j}+n\right)} \cdot \frac{z^{n}}{n !}
$$

In other situations, it is convenient to use the function

$$
{ }_{p} G_{q}\left(\alpha_{1}, \ldots, \alpha_{p} ; \beta_{1}, \ldots, \beta_{q} ; z\right)={ }_{p} F_{q} /\left\{\prod_{j=1}^{q} \Gamma\left(\beta_{j}\right)\right\} .
$$

This is particularly so if any one of the $\beta_{j}$ is a nonpositive integer. The terms of the ${ }_{p} G_{q}$ function involving gamma functions of nonpositive integers may then be suppressed from that function. If we let $\theta$ denote the operator $z D_{z}$, then ${ }_{p} F_{q}$ satisfies the differential equation

$$
\left\{\theta \prod_{j=1}^{q}\left(\theta+\beta_{j}-1\right)-z \prod_{i=1}^{p}\left(\theta+\alpha_{i}\right)\right\} \omega(z)=0
$$

when no $\beta_{j}$ is a nonpositive integer. If some $\beta_{j}$ is a nonpositive integer, the equation (2.4) applies to the ${ }_{p} G_{q}$ function. Logarithmic solutions of (2.4) will only be considered in a special application.

If all of the $\alpha_{i}$ 's and $\beta_{j}$ 's in (2.1) are $>0$, we have the following standard theorems: 
THEOREM 2.1. If $p \leqq q$ and $\alpha_{i}>0, \beta_{j}>0$ for all $i$ and $j$, then

$$
\begin{aligned}
& { }_{p} F_{q}\left(\alpha_{1}, \ldots, \alpha_{p} ; \beta_{1}, \ldots, \beta_{q} ; z t\right)
\end{aligned}
$$

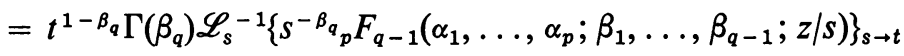

where $\mathscr{L}_{s}^{-1}\{f(s)\}_{s \rightarrow t}$ denotes the inverse Laplace transform of $f(s)$ with $s$ the variable of the transform and the variable of inversion.

THEOREM 2.2. If $p \leqq q+1$ and all $\alpha_{i}$ and $\beta_{j}$ are positive, then

$$
\begin{aligned}
& { }_{p} F_{q}\left(\alpha_{1}, \ldots, \alpha_{p} ; \beta_{1}, \ldots, \beta_{q} ; z\right) \\
& \quad=\frac{1}{\Gamma\left(\alpha_{p}\right)} \int_{0}^{\infty} e^{-\sigma \sigma^{\alpha_{p}-1}{ }_{p-1} F_{q}\left(\alpha_{1}, \ldots, \alpha_{p-1} ; \beta_{1}, \ldots, \beta_{q} ; z \sigma\right) d \sigma .}
\end{aligned}
$$

THEOREM 2.3. Let $p \leqq q+1$ with $\beta_{q}>\alpha_{p}>0$ and no one of $\beta_{1}, \ldots, \beta_{q}$ zero or $a$ negative integer. If $|z|<1$, then

$$
\begin{aligned}
& { }_{p} F_{q}\left(\alpha_{1}, \ldots, \alpha_{p} ; \beta_{1}, \ldots, \beta_{q} ; z\right)=\frac{\Gamma\left(\beta_{q}\right)}{\Gamma\left(\alpha_{p}\right) \Gamma\left(\beta_{q}-\alpha_{p}\right)} \\
& \quad \int_{0}^{1} \sigma^{\alpha_{p}-1}(1-\sigma)^{\beta_{q}-\alpha_{p}-1}{ }_{p-1} F_{q-1}\left(\alpha_{1}, \ldots, \alpha_{p-1} ; \beta_{1}, \ldots, \beta_{q-1} ; z \sigma\right) d \sigma .
\end{aligned}
$$

If $p \leqq q$, the condition $|z|<1$ may be omitted.

We also need results when some of the $\alpha_{i}$ 's or $\beta_{j}$ 's are nonpositive. The following results can be obtained by rearranging the series in (2.2) and using the definition (2.3).

THEOREM 2.4. Let some of the $\beta_{j}$ 's be negative integers or zero with $-m$ the minimum of these. Then

$$
\begin{aligned}
& { }_{p} G_{q}\left(\alpha_{1}, \ldots, \alpha_{p} ; \beta_{1}, \ldots, \beta_{q} ; z\right) \\
& =z^{m+1}\left\{\prod_{i=1}^{p} \frac{\Gamma\left(\alpha_{i}+m+1\right)}{\Gamma\left(\alpha_{i}\right)}\right\}_{p} G_{q}\left(\alpha_{i}+m+1, \ldots, \alpha_{p}+m+1 ; \beta_{1}+m+1, \ldots ; z\right) .
\end{aligned}
$$

THEOREM 2.5. Let some of the $\alpha_{i}^{\prime}$ 's and $\beta_{j}$ 's be negative but not integers. Let $-m+\gamma=\min \left(\alpha_{1}, \ldots, \alpha_{p} ; \beta_{1}, \ldots, \beta_{q}\right)$ where $m$ is a positive integer and $0<\gamma<1$.

Then

$$
\begin{aligned}
{ }_{p} F_{q}\left(\alpha_{1}, \ldots, \alpha_{p} ; \beta_{1}, \ldots, \beta_{q} ; z\right) & =C \sum_{n=0}^{m-1} \frac{\prod_{i=1}^{p} \Gamma\left(\alpha_{i}+n\right)}{\prod_{j=1}^{q} \Gamma\left(\beta_{j}+n\right)} \frac{z^{n}}{n !} \\
& +C C^{*} z^{m}{ }_{p+1} F_{q+1}\left(\alpha_{1}+m, \ldots, \alpha_{p}+m, 1 ; \beta_{1}+m, \ldots, \beta_{q}+m, m+1 ; z\right)
\end{aligned}
$$

where $C=\prod_{j=1}^{q} \Gamma\left(\beta_{j}\right) / \prod_{i=1}^{p} \Gamma\left(\alpha_{i}\right)$ and $C^{*}=\prod_{i=1}^{p} \Gamma\left(\alpha_{i}+m\right) /\left[m ! \prod_{j=1}^{q} \Gamma\left(\beta_{j}+m\right)\right]$.

Observe that the ${ }_{p+1} F_{q+1}$ function in (2.9) reduces to

$$
m \int_{0}^{1}(1-\sigma)^{m-1} F_{q}\left(\alpha_{1}+m, \ldots, \alpha_{p}+m ; \beta_{1}+m, \ldots, \beta_{q}+m ; \sigma z\right) d \sigma
$$

by Theorem 2.3 . 
From these five theorems, we see that the ${ }_{p} F_{q}$ functions can be built from the function ${ }_{0} F_{0}(-;-; z)=e^{z}$ by means of convolutions, Laplace transforms, and inverse Laplace transforms supplemented possibly by polynomials and polynomial multipliers. These results indicate the rather basic importance of the exponential operator $e^{t P(x, D)}$ noted in the Introduction, since all other such hypergeometric operators can be interpreted in terms of this one.

In order to construct examples, let $x=\left(x_{1}, \ldots, x_{n}\right)$ and let $D=\left(D_{1}, \ldots, D_{n}\right)$ with $D_{i} \equiv \partial / \partial x_{i}$. Then define

$$
P(x, D)=\sum_{0 \leqq|\lambda| \leqq m} a_{\lambda}(x) D_{1^{1}}^{\lambda_{1}} \cdots D_{n^{n}}^{\lambda^{n}}
$$

where all $\lambda_{i} \geqq 0$ and $|\lambda|=\lambda_{1}+\cdots+\lambda_{n}$. The conditions required on the coefficients $a_{\lambda}(x)$ for the solvability of the problem

$$
U_{t}(x, t)=P(x, D) U(x, t), \quad U(x, 0)=\phi(x)
$$

will not be considered here. If $P(x, D)$ is an elliptic operator of the form

$$
\sum_{i, j}^{n} a_{i j}(x) D_{i} D_{j}
$$

the reader is referred to [8].

If $n=1$, we have the following interpretations for $u(x, t)=e^{t P(x, D)} \phi(x)$ for special choices of $P(x, D)$ :

(i) $P(x, D)=x^{p} D_{x}$, then $u(x, t)=\phi\left(x e^{t}\right)$ if $p=1$ and $\phi\left[x\left\{1-(p-1) x^{p-1} t\right\}^{-1 / p-1}\right]$ if $p \neq 1$ provided $\phi(x) \in C^{1}$.

(ii) $P(x, D)=D_{x}^{2}$, then $u(x, t)=(4 \pi t)^{-1 / 2} \int_{-\infty}^{\infty} e^{-(x-\xi)^{2} / 4 t} \phi(\xi) d \xi$. If

$$
\phi(x)=\sum_{n=1}^{\infty} a_{n} \sin n \pi x,
$$

then $u(x, t)=\sum_{n=1}^{\infty} a_{n} e^{-n^{2} \pi^{2} t} \sin n \pi x$.

(iii) $P\left(r, D_{r}\right)=D_{r}^{2}+((\mu-1) / r) D_{r}$, then $u(r, t)$ denotes the solution of the radial heat equation ([1], [2]). If $n>1$, we have similar interpretations. The general theory developed in no way depends upon using differential operators for $P$. If $A$ is a linear operator that is $t$ independent, such that one can attach a meaning to $e^{t A} \phi(x)$, then the theory is applicable (subject, of course, to the growth restrictions demanded in those theorems). For example, if $A \phi(x)=\int_{0}^{x} \phi(\xi) d \xi$, then

$$
e^{A t} \phi(x)=\phi(x)+\sqrt{ } t \int_{0}^{x} \frac{I_{1}\left(2(t(x-\xi))^{1 / 2}\right)}{(x-\xi)^{1 / 2}} \phi(\xi) d \xi
$$

Another possible interpretation, for $A$, is a differencing operator.

3. Theorems on related problems. The formulas for the ${ }_{p} F_{q}$ functions in the last section permit us to readily state results on related partial differential equations. 
For, by the formalism of the introduction, we may regard

$$
{ }_{p} F_{q}\left(\alpha_{1}, \ldots, \alpha_{p} ; \beta_{1}, \ldots, \beta_{q} ; t P\right)
$$

as a solution operator for

$$
\left[t D_{t} \prod_{j=1}^{q}\left(t D_{t}+\beta_{j}-1\right)-t P(x, D) \prod_{i=1}^{p}\left(t D_{t}+\alpha_{j}\right)\right] U(x, t)=0 .
$$

Then, if we replace $z$ by $t P(x, D)$ in (2.6), (2.7), (2.8) and (2.9) and $z$ by $P(x, D)$ in (2.5) and formally operate on a suitable data function $\phi(x)$ on both sides of these formulas, the relations obtained may be interpreted as connections between solutions of (3.1). The results obtained can be proved, under suitable restrictions, without resorting to the operator. We state and prove three basic theorems and their corollaries for equations somewhat more general than the one given above.

Let $P(x, D)$ and $Q(x, D)$ denote two partial differential operators, of the type previously described, of orders $l_{1}$ and $l_{2}$, respectively. We often write these as $P$ and $Q$ without changing their meaning. For simplification, let $\theta_{1}\left(q, \beta, t, D_{t}\right)$ $=t D_{t} \prod_{j=1}^{q}\left(t D_{t}+\beta_{j}-1\right)$ where $\beta$ is the vector $\left(\beta_{1}, \ldots, \beta_{q}\right)$, the number of components being dictated by the number $q$ in the symbol $\theta_{1}$. With this notation, $\theta_{1}\left(q-1, \beta, t, D_{t}\right)=t D_{t} \prod_{j=1}^{q-1}\left(t D_{t}+\beta_{j}-1\right)$. Similarly, let

$$
\theta_{2}\left(p, \alpha, t, D_{t}\right)=t \prod_{i=1}^{p}\left(t D_{t}+\alpha_{i}\right)
$$

Let $r=\max \left(p+l_{1}, q+l_{2}\right)$. The differentiability assumptions we introduce are only sufficient ones, and may be weakened for special solution representations.

LEMMA 3.1. Let $p \leqq q$ and assume that all $\alpha_{i}$ and $\beta_{j}$ are positive. Let $u(x, t) \in C^{r}$ in $x$ and $t$ for $t>0$ such that $u(x, t)$ and all of its derivatives through order $r$ are bounded. If $\lim _{t \rightarrow 0} u(x, t)$ exists and is finite, then the function

$$
W(x, t)=\int_{0}^{\infty} e^{-\sigma \sigma^{\alpha}-1} u(x, t \sigma) d \sigma
$$

satisfies the relation

$$
\begin{aligned}
& {\left[Q(x, D) \theta_{1}\left(q, \beta, t, D_{t}\right)-P(x, D) \theta_{2}\left(p, \alpha, t, D_{t}\right)\right] W(x, t)} \\
& \quad=\int_{0}^{\infty} e^{-\sigma_{\sigma^{\alpha}}-1}\left[Q(x, D) \theta_{1}\left(q, \beta, t \sigma, D_{t \sigma}\right)\right. \\
& \left.\quad-P(x, D) \theta_{2}\left(p-1, \alpha, t \sigma, D_{t \sigma}\right)\right] u(x, t \sigma) d \sigma .
\end{aligned}
$$

Proof. We first observe that

$$
\begin{aligned}
\left(t D_{t}+\alpha_{p}\right) W(x, t) & =\int_{0}^{\infty} e^{-\sigma} \sigma^{\alpha_{p}-1}\left\{t D_{t} u(x, \sigma t)+\alpha_{p} u(x, \sigma t)\right\} d \sigma \\
& =\int_{0}^{\infty} e^{-\sigma \sigma^{\alpha_{p}-1}\left\{\sigma D_{\sigma} u(x, t \sigma)+\alpha_{p} u(x, t \sigma)\right\} d \sigma}
\end{aligned}
$$


since $t D_{t} u(x, t \sigma)=\sigma D_{\sigma} u(x, t \sigma)$. An integration of the first member of this by parts (invoking the existence and finiteness of $\lim _{t \rightarrow 0+} u(x, t)$ ) gives $\left(t D_{t}+\alpha_{p}\right) W(x, t)$ $=\int_{0}^{\infty} e^{-\sigma} \sigma^{\alpha} p u(x, t \sigma) d \sigma$. Then

$$
\begin{aligned}
& P(x, D) \theta_{2}\left(p, \alpha, t, D_{t}\right) W(x, t)=P(x, D) \theta_{2}\left(p-1, \alpha, t, D_{t}\right) \int_{0}^{\infty} e^{-\sigma} \sigma^{\alpha_{p}} u(x, t \sigma) d \sigma \\
& =\int_{0}^{\infty} e^{-\sigma} \sigma^{\alpha_{p}}\left[P(x, D) \theta_{2}\left(p-1, \alpha, t, D_{t}\right)\right] u(x, t \sigma) d \sigma \\
& =\int_{0}^{\infty} e^{-\sigma \sigma^{\alpha} \alpha^{-1}}\left[P(x, D) \theta_{2}\left(p-1, \alpha, t \sigma, D_{t \sigma}\right)\right] u(x, t \sigma) d \sigma
\end{aligned}
$$

since $t D_{t} u(x, t \sigma)=\sigma D_{\sigma} u(x, t \sigma)=(t \sigma) D_{t \sigma} u(x, t \sigma)$. Similarly

$\left[Q(x, D) \theta_{1}\left(q, \beta, t, D_{t}\right)\right] W(x, t)=\int_{0}^{\infty} e^{-\sigma} \sigma^{\alpha_{p}-1}\left[Q(x, D) \theta_{1}\left(q, \beta, t \sigma, D_{t \sigma}\right)\right] u(x, t \sigma) d \sigma$.

The stated result follows from these two computations.

THEOREM 3.1. Let $u(x, t)$ be a solution of the equation

$$
\left[Q(x, D) \theta_{1}\left(q, \beta, t, D_{t}\right)-P(x, D) \theta_{2}\left(p-1, \alpha, t, D_{t}\right)\right] u(x, t)=0
$$

satisfying the conditions of Lemma 3.1. Let $\alpha_{p}>0$. Then the function

$$
V(x, t)=\left\{\Gamma\left(\alpha_{p}\right)\right\}^{-1} \int_{0}^{\infty} e^{-\sigma} \sigma^{\alpha_{p}-1} u(x, t \sigma) d \sigma
$$

is a solution of the equation (3.4) with $p$ replaced by $p+1$. If $\lim _{t \rightarrow 0+} u(x, t)=\phi(x)$, with $\phi(x)$ bounded and continuous, then $\lim _{t \rightarrow 0+} V(x, t)=\phi(x)$.

Proof. The fact that $V(x, t)$ satisfies (3.4), with $p$ replaced by $p+1$, follows from Lemma 3.1. In order to show that the initial condition holds, set

$$
W(x, t)=V(x, t)-\phi(x)=\left\{\Gamma\left(\alpha_{p}\right)\right\}^{-1} \int_{0}^{\infty} e^{-\sigma^{\alpha_{p}-1}\{u(x, t \sigma)-\phi(x)\} d \sigma .}
$$

Since $u(x, t)$ and $\phi(x)$ are bounded, there exists $M>0$ such that

$$
\left[\Gamma\left(\alpha_{p}\right)\right]^{-1}|u(x, t \sigma)-\phi(x)|<M .
$$

Given $\varepsilon>0$, choose $R>0$ such that $\int_{R}^{\infty} e^{-\sigma^{\alpha_{p}-1}} d \sigma<\Gamma\left(\alpha_{p}\right) \varepsilon /\{2 M\}$. Then select $t$ so small that

$$
\left[\Gamma\left(\alpha_{p}\right)\right]^{-1} \int_{0}^{R} e^{-\sigma \sigma^{\alpha_{p}-1}}|u(x, t \sigma)-\phi(x)| d \sigma<\varepsilon / 2 .
$$

With these relations, it follows that $|W(x, t)|<\varepsilon$ and proves that

$$
\lim _{t \rightarrow 0+} V(x, t)=\phi(x) \text {. }
$$

An examination of the integral (3.5) gives the following corollaries almost immediately: 
COROLlary 3.1a. Let $S$ denote a cylindrical surface defined by an equation of the form $h(x)=0$, and let $B(x, D)$ be a nontangential ( $t$ independent) boundary operator on $S$, of order <r. If $\left.B(x, D) u(x, t)\right|_{S}=f(x, t), f$ bounded, then $\left.B(x, D) V(x, t)\right|_{S}$ $=g(x, t)$ with $g(x, t)=\left\{\Gamma\left(\alpha_{p}\right)\right\}^{-1} \int_{0}^{\infty} e^{-\sigma} \sigma^{\alpha_{p}-1} f(x, t \sigma) d \sigma$.

COROLlaRY 3.2b. If $u(x, t)$ is an eigenfunction for the problem defined by equation (3.4) and the condition $\left.B(x, D) u(x, t)\right|_{S}=0$, then the function $V(x, t)$ defined by (3.5) is an eigenfunction for the problem defined by the equation (3.4) with $p$ replaced by $p+1$ and satisfying the condition $\left.B(x, D) V(x, t)\right|_{s}=0$.

REMARK 1. An examination of the proof of Lemma 3.1 shows that it depends in no essential way on the forms of the operators $P$ and $Q$ (except in requiring $u(x, t)$ to have a sufficient number of derivatives permitting the interchange of the orders of the operations). The key steps involved arguments pertaining to the second variable in $u(x, t \sigma)$, such as integration by parts, and using such relations as $t D_{t} u(x, t \sigma)=\sigma D_{\sigma} u(x, t \sigma)=(t \sigma) D_{(t \sigma)} u(x, t \sigma)$. This shows that the proof of this lemma is, basically, the same as the proof of Theorem 2.2, and indicates why $P$ and $Q$ can be treated as parameters. This observation points out why our formalism leads to correct results. We will make use of this remark in the next two theorems.

LEMMA 3.2. Let all $\alpha_{i}$ and $\beta_{j}$ be positive with $\beta_{q}>1$. Let $U(x, t) \in C^{r}$ and assume that $U(x, t)$ and its derivatives through order $r$ are bounded. Assume that

exists and is finite and let

$$
\lim _{t \rightarrow 0+} U(x, t)
$$

$$
W(x, t)=\int_{0}^{\infty} e^{-\sigma^{\beta_{q}-1}} U(x, t \sigma) d \sigma
$$

Then

$$
\begin{aligned}
& {\left[Q \theta_{1}\left(q-1, \beta, t, D_{t}\right)-P \theta_{2}\left(p, \alpha, t, D_{t}\right)\right] W(x, t)} \\
& \quad=\int_{0}^{\infty} e^{-\sigma_{\sigma_{q}}-2}\left[Q \theta_{1}\left(q, \beta, t \sigma, D_{t \sigma}\right)-P \theta_{2}\left(p, \alpha, t \sigma, D_{t \sigma}\right)\right] U(x, t \sigma) d \sigma .
\end{aligned}
$$

Proof. The proof is essentially the same as the proof of Lemma 3.1. The key step is contained in the following chain of relations:

$$
\begin{aligned}
\int_{0}^{\infty} e^{-\sigma_{\sigma^{\beta_{q}-2}}} & {\left[Q \theta_{1}\left(q, \beta, t \sigma, D_{t \sigma}\right)\right] U(x, t \sigma) d \sigma } \\
& =\int_{0}^{\infty} e^{-\sigma \sigma^{\beta_{q}-2}}\left[Q \theta_{1}\left(q, \beta, t, D_{t}\right)\right] U(x, t \sigma) d \sigma \\
& =Q \theta_{1}\left(q-1, \beta, t, D_{t}\right) \int_{0}^{\infty} \sigma^{\beta_{q}-2} e^{-\sigma}\left[t D_{t}+\beta_{q}-1\right] U(x, t \sigma) d \sigma \\
& =Q \theta_{1}\left(q-1, \beta, t, D_{t}\right) \int_{0}^{\infty} \sigma^{\beta_{q}-2} e^{-\sigma}\left[\sigma D_{\sigma} U+\left(\beta_{q}-1\right) U\right] d \sigma
\end{aligned}
$$


An integration by parts, using the finiteness of $U(x, 0)$, shows that the integral in the last member of (3.8) reduces to $W(x, t)$. The remainder of the proof follows readily.

THEOREM 3.2. Let all $\alpha_{i}$ and $\beta_{j}$ be positive with $\beta_{q}>1$. Let $u(x, t)$ be a solution of the equation

$$
\left[Q \theta_{1}\left(q-1, \beta, t, D_{t}\right)-P \theta_{2}\left(p, \alpha, t, D_{t}\right)\right] u(x, t)=0
$$

for $t>0$. Let

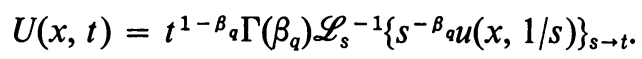

If $U(x, t)$ exists and $U(x, t) \in C^{r}$ and $U$ and its derivatives through order $r$ are bounded, then

$$
\left[Q \theta_{1}\left(q, \beta, t, D_{t}\right)-P \theta_{2}\left(p, \alpha, t, D_{t}\right)\right] U(x, t)=0
$$

for $t>0$. If $u(x, 1 / s)$ is bounded and analytic in $s$ for $\operatorname{Re}(s)>k$ for some $k>0$, then $\lim _{t \rightarrow 0+} U(x, t)=\phi(x)$.

Proof. The conditions on $U(x, t)$ permit inverting (3.10) to give

$$
u(x, t)=\left\{\Gamma\left(\beta_{q}\right)\right\}^{-1} \int_{0}^{\infty} e^{-\sigma^{\beta_{q}-1} U(x, t \sigma) d \sigma .}
$$

By Lemma 3.2, as applied to $u(x, t)$, it follows that the integral in the right member of (3.7) vanishes. By the uniqueness theorem of Lerch, it follows that the integrand in (3.7) must vanish. (Make the change of variables $t \sigma=\xi$ to see that this theorem is applicable with $t$ the variable of transformation.) This proves (3.11). That the initial condition holds follows by a standard argument.

REMARK 2. The standard inversion theorems for the Laplace transform are too severe to assert directly the existence and differentiability of $U(x, t)$ in (3.10). In special cases, the inversion in (3.10) can be carried out explicitly, and conditions on $U$ can be checked directly.

COROLlaRY 3.1. Let $\beta_{q}^{*}>\beta_{q}>1$ and let $U\left(x, t ; \beta_{q}\right)$ be a solution of $(3.11)$ defined by (3.10). If $U\left(x, t ; \beta_{q}^{*}\right)$ is obtained by replacing $\beta_{q}$ by $\beta_{q}^{*}$ in (3.10), then

$$
U\left(x, t ; \beta_{q}^{*}\right)=\frac{1}{B\left(\beta_{q}, \beta_{q}^{*}-\beta_{q}\right)} \int_{0}^{1}(1-\sigma)^{\beta_{q}^{*}-\beta_{q}-1}{\sigma^{\beta_{q}-1}}^{-1}\left(x, t \sigma ; \beta_{q}\right) d \sigma
$$

and $U\left(x, t ; \beta_{q}^{*}\right)$ satisfies $(3.11)$ with $\beta_{q}$ replaced by $\beta_{q}^{*}$.

Proof. This follows readily by applying the convolution theorem for Laplace transforms to the formula of the type (3.10) defining $U\left(x, t ; \beta_{q}^{*}\right)$.

COROLLARY 3.2. Let $S$ be a cylindrical surface defined by an equation of the form $h(x)=0$ and let $B(x, D)$ be a nontangential boundary operator of order $<r$. If 
$u(x, t)$ is a solution of (3.9) satisfying the condition $\left.B(x, D) u(x, t)\right|_{s}=f(x, t), f(x, t)$ bounded, then the function $U(x, t)$ defined by (3.10) satisfies the condition

$$
\left.B(x, D) U(x, t)\right|_{s}=g(x, t)
$$

provided $g(x, t)=t^{1-\beta_{q}} \Gamma\left(\beta_{q}\right) \mathscr{L}_{s}^{-1}\left\{s^{-\beta_{q}} f(x, 1 / s)\right\}_{s \rightarrow t}$. In particular, eigensolutions of (3.9) transform into eigensolutions of (3.11) by the relation (3.10).

THEOREM 3.3. Let $p \leqq q+1, \beta_{q}>\alpha_{p}>0$ and no one of the numbers $\beta_{1}, \ldots, \beta_{q-1}$ zero or a negative integer. Let $u(x, t) \in C^{r-1}$ satisfy the equation

$$
\left[Q \theta_{1}\left(q-1, \beta, t, D_{t}\right)-P \theta_{2}\left(p-1, \alpha, t, D_{t}\right)\right] u(x, t)=0
$$

for $t>0$ such that $\lim _{t \rightarrow 0} u(x, t)=\phi(x)$ with $\phi(x)$ continuous. Let $U(x, t)$ be defined by

$$
U(x, t)=\frac{\Gamma\left(\beta_{q}\right)}{\Gamma\left(\alpha_{p}\right) \Gamma\left(\beta_{q}-\alpha_{p}\right)} \int_{0}^{1} \sigma^{\alpha_{p}-1}(1-\sigma)^{\beta_{q}-\alpha_{p}-1} u(x, t \sigma) d \sigma .
$$

Then $U(x, t)$ satisfies

$$
\left[Q \theta_{1}\left(q, \beta, t, D_{t}\right)-P \theta_{2}\left(p, \alpha, t, D_{t}\right)\right] U(x, t)=0
$$

for $t>0$ and $\lim _{t \rightarrow 0+} U(x, t)=\phi(x)$.

Proof. The proof that the initial condition holds is trivial. The Remark 1 permits us to prove that $U(x, t)$ satisfies (3.14). We need only verify that $U(x, t) \in C^{r}$ for $t>0$. We verify this if $\beta_{q}-\alpha_{p}>1$. If we let

$$
\omega(x, t)=\int_{0}^{1} \sigma^{\alpha_{p}-1}(1-\sigma)^{\beta_{q}-\alpha_{p}-1} u(x, t \sigma) d \sigma .
$$

Then

$$
\begin{aligned}
\omega_{t}(x, t) & =\int_{0}^{1} \sigma^{\alpha_{p}-1}(1-\sigma)^{\beta_{q}-\alpha_{p}-1} u_{t}(x, t \sigma) d \sigma \\
& =\int_{0}^{1} \sigma^{\alpha_{p}}(1-\sigma)^{\beta_{q}-\alpha_{p}-1} u_{\sigma}(x, t \sigma) d \sigma
\end{aligned}
$$

since $u_{t}(x, t \sigma)=(\sigma / t) u_{\sigma}(x, t \sigma)$. An integration of this last integral by parts gives

$$
\left.\omega_{t}(x, t)=-t^{-1} \int_{0}^{1}(1-\sigma)^{\beta_{q}-\alpha_{p}-2}{\sigma^{\alpha_{p}}-1}^{1} \alpha_{p}+\left(1-\beta_{q}\right) \sigma\right] u(x, t \sigma) d \sigma .
$$

Since $u(x, t) \in C^{r-1}$, the result follows.

Corollaries on boundary value problems can be proved that are analogous to Corollaries 3.1a, 3.1b, and 3.2. We omit the statement of these results.

4. Theorems on index shifting. The theorems of the preceding section involved shifts on the integers $p$ and $q$. We now give results relating to the parameters $\alpha_{i}$ and $\beta_{j}$. In some of these theorems, we must select $Q(x, D)=1$. 
THEOREM 4.1. Let some of the numbers $\alpha_{1}, \ldots, \alpha_{p}$ be negative integers or zero and let $-m$ be the largest of these nonpositive integers. If none of the $\beta_{j}$ are zero or negative integers, then a solution of

$$
\left[\theta_{1}\left(q, \beta, t, D_{t}\right)-P(x, D) \theta_{2}\left(p, \alpha, t, D_{t}\right)\right] U(x, t)=0,
$$

$U(x, 0)=\phi(x)$ is given by

$$
U(x, t)=\phi(x)+\sum_{n=1}^{m}\left\{\frac{\prod_{i=1}^{p}\left(\alpha_{i}\right)_{n}}{\prod_{j=1}^{q}\left(\beta_{j}\right)_{n}}\right\} \frac{t^{n}}{n !} P^{n}(x, D) \phi(x)
$$

provided that $\phi(x) \in C^{l_{1}(m+1)}$.

Proof. This follows directly from (2.1), Remark 1 , and by regarding $P(x, D)$ as a parameter. The condition on $\phi(x)$ suffices for (4.2) to define a classical solution.

THEOREM 4.2. Let some of the $\alpha_{i}$ 's and $\beta_{j}$ 's be negative numbers none of which are integers. Let $-m+\gamma=\min \left(\alpha_{1}, \ldots, \alpha_{p} ; \beta_{1}, \ldots, \beta_{q}\right)$ with $m a$ positive integer and $0<\gamma<1$. Let $\phi(x) \in C^{m l_{1}+r}$. Then if $u(x, t)$ satisfies

$$
\left[t D_{t} \prod_{j=1}^{q}\left(t D_{t}+\beta_{j}+m-1\right)-t P \prod_{i=1}^{p}\left(t D_{t}+\alpha_{i}+m\right)\right] u(x, t)=0,
$$

$u(x, 0)=\phi(x)$, it follows that a solution of

$$
\left[\theta_{1}\left(q, \beta, t, D_{t}\right)-P \theta_{2}\left(p, \alpha, t, D_{t}\right)\right] U(x, t)=0,
$$

$U(x, 0)=\phi(x)$ is given by

$$
\begin{aligned}
U(x, t)= & \sum_{n=0}^{m-1}\left\{\frac{\prod_{i=1}^{p}\left(\alpha_{i}\right)_{n}}{\prod_{j=1}^{q}\left(\beta_{j}\right)_{n}}\right\} \frac{t^{n}}{n !} P^{n}(x, D) \phi(x) \\
& +m c c^{*} t^{m} P^{m}(x, D) \int_{0}^{1}(1-\sigma)^{m-1} u(x, t \sigma) d \sigma
\end{aligned}
$$

where $C$ and $C^{*}$ are described in $\$ 2$.

The proof follows by Remark 1 .

For the remaining theorems in this section, we need the following elementary result:

$$
\left(t D_{t}+\gamma\right)\left(t^{m} U(x, t)\right)=t^{m}\left(t D_{t}+\gamma+m\right) U(x, t) .
$$

Then we have:

THEOREM 4.3. Let $U(x, t)$ be a solution of the equation

$$
\left[Q t D_{t}\left(t D_{t}+\beta_{l}\right) \prod_{j=1 ; j \neq l}^{q}\left(t D_{t}+\beta_{j}-1\right)-P \theta_{2}\left(p, \alpha, t, D_{t}\right)\right] U(x, t)=0
$$


$1 \leqq l \leqq q$. Then the function $V(x, t)=\left(t D_{t}+\beta_{l}\right) U(x, t)$ satisfies the equation

$$
\left[Q \theta_{1}\left(q, \beta, t, D_{t}\right)-P \theta_{2}\left(p, \alpha, t, D_{t}\right)\right] V(x, t)=0
$$

if $U(x, t) \in C^{r+1}$. If $\lim _{t \rightarrow 0} U(x, t)=\phi(x)$ and $\beta_{l} \neq 0$,

$$
\lim _{t \rightarrow 0} \frac{1}{\beta_{l}} V(x, t)=\phi(x) .
$$

Proof. The factors of the form $\left(t D_{t}+\gamma\right)$ commute with one another. If we substitute the expression for $V(x, t)$ into (4.5), the first term there becomes

$$
\left(t D_{t}+\beta_{l}-1\right)\left\{Q\left(t D_{t}\right)\left(t D_{t}+\beta_{l}\right) \prod_{j=1 ; j \neq l}^{q}\left(t D_{t}+\beta_{j}-1\right)\right\} U
$$

while the second term reduces to

$$
\left(t D_{t}+\beta_{l}-1\right)\left\{t P \theta_{2}\right\} U
$$

by (4.3). The result follows by subtracting (4.7) from (4.6) and noting (4.4).

THEOREM 4.4. Let $u(x, t)$ be a solution of the equation

$$
\left[Q \theta_{1}\left(q, \beta, t, D_{t}\right)-P \theta_{2}\left(p, \alpha, t, D_{t}\right)\right] u(x, t)=0
$$

with $u \in C^{r+1}$. If $1 \leqq l \leqq p$, then the function $U(x, t)=\left(t D_{t}+\alpha_{l}\right) u(x, t)$ satisfies the equation

$$
\left[Q \theta_{1}\left(q, \beta, t, D_{t}\right)-P t\left(t D_{t}+\alpha_{l}+1\right) \prod_{i=1 ; j \neq l}^{p}\left(t D_{t}+\alpha_{i}\right)\right] U(x, t)=0 .
$$

If $\alpha_{l} \neq 0$ and $\lim _{t \rightarrow 0} u(x, t)=\phi(x)$,

$$
\lim _{t \rightarrow 0}\left(U(x, t) / \alpha_{l}\right)=\phi(x) .
$$

Proof. The same as in the proof of Theorem 4.3.

From (4.3), it follows that

$$
\left[Q \theta_{1}\left(q, \beta, t, D_{t}\right)-P \theta_{2}\left(p, \alpha, t D_{t}\right)\right]\left(t^{m} U(x, t)\right)
$$

$$
=t^{m}\left\{Q\left(t D_{t}+m\right) \prod_{j=1}^{q}\left(t D_{t}+\beta_{j}+m-1\right)-t P \prod_{i=1}^{p}\left(t D_{t}+\alpha_{i}+m\right)\right\} U(x, t) .
$$

If we select $m=1-\beta_{l}$ for $l=1,2, \ldots, q$, it follows that the operator in the right member of (4.8) reduces to one of those in the class we have been considering. From this we conclude

THEOREM 4.5. Let $U(x, t)$ be a solution of the differential equation

$$
\begin{array}{r}
{\left[Q\left(t D_{t}\right)\left\{t D_{t}+\left(2-\beta_{l}\right)-1\right\} \prod_{j=1 ; j \neq l}^{q}\left(t D_{t}+\beta_{j}-\beta_{l}\right)-t P \prod_{i=1}^{p}\left(t D_{t}+\alpha_{i}+1-\beta_{l}\right)\right]} \\
\times U(x, t)=0 .
\end{array}
$$


Then the function $V(x, t)=t^{1-\beta_{l}} U(x, t)$ satisfies

$$
\left[Q \theta_{1}\left(q, \beta, t, D_{t}\right)-P \theta_{2}\left(p, \alpha, t, D_{t}\right)\right] V(x, t)=0
$$

for $l=1,2, \ldots, q$.

5. Applications of related problems. In this section, we give some examples of problems indicating how the connections obtained in $\$ \$ 3$ and 4 can be applied in particular cases. The relation of our results to those of A. Weinstein, for the Euler-Poisson-Darboux equation, will be discussed in $\S 6$.

EXAMPLE 1. Let $U(x, t)$ satisfy the problem

$$
t \frac{\partial^{2} U}{\partial x \partial t}(x, t)=\frac{\partial U}{\partial t}(x, t)-\frac{\partial U}{\partial x}(x, t), \quad U(x, 0)=\phi(x)
$$

with $\phi(x) \in C^{1}$. This second order hyperbolic equation degenerates, to a first order hyperbolic equation, where the data is assigned. It is a curiosity, in this case, that the right member of the equation in (5.1) contains all of the terms of the related equation. The stated equation can be written in the form $\left(1-t D_{x}\right) U_{t}=D_{x} U$, and has the solution operator series ${ }_{1} F_{0}\left(1 ;-; t D_{x}\right)$. By Theorem 3.1 , the problem related to $(5.1)$ is $u_{t}=u_{x}, u(x, 0)=\phi(x)$ which has the solution $\phi(x+t)$. By Theorem 3.1 , it follows that a solution of (5.1) is given by

$$
U(x, t)=\int_{0}^{\infty} e^{-\sigma} \phi(x+t \sigma) d \sigma=t^{-1} \int_{x}^{\infty} e^{(x-\sigma) / t} \phi(\sigma) d \sigma .
$$

If $|\phi(x)|<M e^{\alpha x}$ for some $\alpha>0$ as $x \rightarrow \infty$, then the integral exists if $0<t<1 / \alpha$. A standard argument suffices to show that $\lim _{t \rightarrow 0+} U(x, t)=\phi(x)$ for this type of data.

Next, replace $D_{x}$ in equation (5.1) by $x D_{x}$ and recall that $e^{t x D_{x}} \phi(x)=\phi\left(x e^{t}\right)$. Then

$$
U(x, t) \int_{0}^{\infty} e^{-\sigma} \phi\left(x e^{\sigma t}\right) d t .
$$

If $\phi(x)$ is bounded, the integral in (5.3) exists. Its derivative may fail to exist however. For if we take $\phi(x)=\sin \left(e^{x}\right)$, then $\phi\left(x e^{\sigma t}\right)=\sin \left\{e^{x e^{\sigma t}}\right\}$ and $\phi_{x}\left(x e^{\sigma t}\right)$ $=e^{\sigma t} e^{x e^{\sigma t}} \cos \left\{e^{x e^{\sigma t}}\right\}$. In this case, the differentiated integral in (5.3) fails to converge if $x>0$ and $t>0$.

EXAMPLE 2. Consider the equation $t^{4} U_{t t}(x, t)-D_{x}^{2} U(x, t)=0$. The associated ordinary differential equation $t^{4} Y_{t t}-a^{2} Y=0$, obtained by replacing $D_{x}$ by a constant $a$, has a regular singular point at $t=\infty$. It seems plausible that we could assign a value for $\lim _{t \rightarrow \infty} U(x, t)$ in advance, say $\phi(x)$. With the change of variables $t=\xi^{-1}$, the ordinary equation becomes $\xi Y_{\xi \xi}+2 Y_{\xi}-a^{2} \xi Y=0$ which has the normalized series solution ${ }_{0} F_{1}\left(-; 3 / 2 ; a^{2} \xi^{2} / 4\right)$ near $\xi=0$. The solution operator related to the stated partial differential equation then is

$$
{ }_{0} F_{1}\left(-; 3 / 2 ; D_{x}^{2} / 4 t^{2}\right)=t \Gamma(3 / 2) \mathscr{L}_{s}^{-1}\left\{s^{-3 / 2} e^{D_{x}^{2} / 4 s}\right\}_{s \rightarrow t^{-2}}
$$


(by Theorem 2.5). If we formally operate on $\phi(x)$ on both sides of this operator relation, it results that

$$
U(x, t)=t \Gamma(3 / 2) \mathscr{L}_{s}^{-1}\left\{s^{-3 / 2} u(x, 1 / 4 s)\right\}_{s \rightarrow t^{-2}}
$$

where $u(x, t)$ is the solution of the heat problem $u_{t}=D_{x}^{2} u, u(x, 0)=\phi(x)$. Carrying out this last inversion (by the results of [5]),

$$
U(x, t)=\frac{t}{2} \int_{x-t^{-1}}^{x+t-1} \phi(\sigma) d \sigma .
$$

It is easy to check that this $U(x, t)$ has the desired properties if $t>0$. If

then $\lim _{t \rightarrow 0+} U(x, t)=0$.

$$
\phi(x) \in L^{1}(-\infty, \infty)
$$

EXAMPLE 3. In a recent book [9, p. 182], the following problem was considered:

$$
U_{t t}(x, t)=t^{m} U_{x x}+v t^{m / 2-1} U_{x}, \quad m \geqq 2,
$$

with $U(x, 0)=\phi(x), U_{t}(x, 0)=\psi(x)$ for the range $|\nu|<m / 2$. We show that the results of this paper permit obtaining solutions without imposing this restriction on $\nu$. We take $\psi(x)=0$ in our analysis.

Under the change of variables $\xi=(4 /(m+2)) t^{(m+2) / 2}$, the problem (5.4) becomes

$$
\begin{aligned}
\xi U_{\xi \xi}+\frac{m}{m+2} U_{\xi} & =\frac{1}{4} \xi U_{x x}+\frac{\nu}{m+2} U_{x} \\
U(x, 0) & =\phi(x) .
\end{aligned}
$$

The further transformation $y=x-\frac{1}{2} \xi, z=\xi$ permits us to reduce this to the problem

$$
\left[z D_{z}\left(z D_{z}+\frac{m}{m+2}-1\right)-z D_{y}\left(z D_{z}+\frac{m-2 \nu}{2 m+4}\right)\right] U=0
$$

$$
U(y, 0)=\phi(y) \text {. }
$$

This form of the problem fits into our theory. After solving (5.6) by that theory, we obtain successively, $U(y, z), U(x, \xi)$, and then $U(x, t)$. To simplify expressions, set $\alpha=(m-2 v) /(2 m+4)$ and $\beta=m /(m+2)$. We will not treat the case $\alpha \leqq 0$, since results follow readily from Theorems 4.1 and 4.2. We distinguish four cases.

Case $1.0<\alpha<\beta$ or $|\nu|<m / 2$. Then Theorem 3.3 is applicable with $u_{z}=u_{y}$, $u(y, 0)=\phi(y)$ as the related problem. This has the solution $u(y, z)=\phi(y+z)$ so that, by (3.13)

$$
U(y, z)=\frac{\Gamma(\beta)}{\Gamma(\alpha) \Gamma(\beta-\alpha)} \int_{0}^{1} \sigma^{\alpha-1}(1-\sigma)^{\beta-\alpha-1} \phi(y+z \sigma) d \sigma
$$

A return to the original variables gives the solution in [9].

Case 2. $\alpha=\beta$ or $\nu=-m / 2$. The equation in (5.6) reduces to $U_{z}=U_{y}$. The solution of (5.6) then is $\phi(y+z)$. 
Case 3. $\alpha-\beta=k$ a positive integer or $\nu=-\frac{1}{2} m-k(m+2), k=1,2, \ldots$ Theorem 4.4 is applicable to give

$$
U(y, z)=\left(1+\frac{z}{\alpha} D_{z}\right)\left(1+\frac{z}{\alpha+1} D_{z}\right) \cdots\left(1+\frac{z}{\alpha+k-1} D_{z}\right) \phi(y+z) .
$$

Case 4. $\alpha>\beta$ with $k$ the least positive integer such that $\beta+k>\alpha$ or

$$
-\frac{1}{2} m-k(m+2)<\nu<-\frac{1}{2} m-(k-1)(m+2) .
$$

Let $U^{\alpha, \beta+k}(y, z)$ be the solution as defined in Case 1 with $\beta$ replaced by $\beta+k$. Theorem 4.3 then applies to give

$$
U^{\alpha, \beta}(y, z)=\left(1+\frac{z}{\beta} D_{z}\right)\left(1+\frac{z}{\beta+1} D_{z}\right) \cdots\left(1+\frac{z}{\beta+k-1} D_{z}\right) U^{\alpha, \beta+k}(y, z) .
$$

Example 4. The Beltrami Problem. Theorem 3.1 can be extended to apply to problems involving equations slightly different from the ones treated there. Under the transformation $t=\xi^{-1}$, it follows that $t D_{t}=-\xi D_{\xi}$ and that the equations of the type we have been considering transform into the form

$$
\left[(-1)^{p} P \prod_{i=1}^{p}\left(\xi D_{\xi}-\alpha_{i}\right)+(-1)^{q} Q \xi \prod_{j=1}^{q+1}\left(\xi D_{\xi}+1-\beta_{j}\right)\right] U=0
$$

if one takes $\beta_{q+1}=1$. Solutions of this equation can be constructed from solutions of an equation obtained by omitting one of the factors $\xi D_{\xi}+1-\beta_{l}(l \neq q+1)$ by a theorem entirely similar to Theorem 3.1. The only restriction is that $1-\beta_{l}>0$. The relating of the solutions of these at $\xi=0$ corresponds to the relating of the solutions of the nontransformed equations at $t=\infty$. This minor extension is needed to treat Dirichlet type problems.

Consider the Beltrami problem

$$
U_{t t}(x, t)+(a / t) U_{t}(x, t)+\Delta_{n} U(x, t)=0, \quad U(x, 0)=\phi(x)
$$

where $\Delta_{n}$ is the Laplacian operator. Under the change of variables $t=\xi^{-1 / 2}$, the equation in (P1) transforms into

$$
\left\{\frac{1}{4} \Delta_{n}+\xi\left(\xi D_{\xi}\right)\left(\xi D_{\xi}+(1-a) / 2\right)\right\} U=0 .
$$

Similarly, the change of variables $t=1 /(4 \xi)$ in the heat problem

$$
u_{t}(x, t)=\Delta_{n} u(x, t), \quad u(x, 0)=\phi(x)
$$

leads to the equation $\left[\frac{1}{4} \Delta_{n}+(\xi)\left(\xi D_{\xi}\right)\right] u \doteq 0$. The extended version of Theorem 3.1 then formally shows that

$$
U\left(x, \xi^{-1 / 2}\right)=\frac{1}{\Gamma((1-a) / 2)} \int_{0}^{\infty} e^{-\sigma_{\sigma}-(a+1) / 2} u(x, 1 /(4 \sigma \xi)) d \sigma
$$

provided $a<1$. A return to the original variable $t$ gives the formula

$$
U(x, t)=\frac{t^{1-a}}{\Gamma((1-a) / 2)} \int_{0}^{\infty} e^{-\sigma t^{2}} \sigma^{-(a+1) / 2} u(x, 1 /(4 \sigma)) d \sigma .
$$


It is easy to show that if $u(x, t)$ is a bounded solution of (5.9) corresponding to bounded and continuous $\phi(x)$, then $U(x, t)$ is a solution of (5.8). Observe that the restriction $a<1$ required for the correct posing of the problem (5.8) arises in a rather natural way by this method. If we introduce

$$
u(x, t)=(4 \pi t)^{-n / 2} \exp \left[-\sum_{i=1}^{n}\left(x_{i}-\xi_{i}\right)^{2} / 4 t\right],
$$

the above formula gives the fundamental solution for (5.8). This result was obtained earlier by J. B. Diaz [13].

6. The Euler-Poisson-Darboux equation. It has been shown in [6] that the Euler-Poisson-Darboux initial value problem

$$
U_{t t}+(a / t) U_{t}=P(x, D) U, \quad U(x, 0)=\phi(x), \quad U_{t}(x, 0)=0
$$

has the solution operator $O(t, P)$ given by

$$
O(t, P)=\frac{2^{(a-1) / 2} \Gamma((a+1) / 2) t^{(1-a) / 2}}{P^{(a-1) / 4}} I_{(a-1) / 2}\left(t P^{1 / 2}\right) .
$$

This operator can be more conveniently expressed in the form

$$
O(t, P)={ }_{0} F_{1}\left(-;(a+1) / 2 ; \frac{1}{4} t^{2} P\right) .
$$

As we have seen, this operator is meaningful if $(a+1) / 2 \neq-m, m=0,1,2, \ldots$ The cases with equality require use of the operator

$$
\frac{1}{\Gamma((a+1) / 2)} O(t, P) \text {. }
$$

With the change of variables $\xi=\frac{1}{4} t^{2}$, the equation in (6.1) becomes

$$
\left\{\xi D_{\xi}\left(\xi D_{\xi}+[(a+1) / 2]-1\right)-\xi P\right\} U(x, \xi)=0
$$

and fits into our theory. We now show how our results specialize to give many of the relations obtained by A. Weinstein. (See [11], [12]. The last of these contains numerous other references.)

Theorem 4.5 asserts that if $f(x, \xi)$ satisfies (6.2) with $a$ replaced by $2-a$, then the function $g(x, \xi)=\xi^{1-(a+1) / 2} f(x, \xi)$ satisfies (6.2). If we denote a solution of the equation in (6.1) corresponding to $a$ by $U^{(a)}(x, t)$, then this last relation, after a return to the $t$ variable, states that $U^{(a)}(x, t)=t^{1-a} U^{(2-a)}(x, t)$, one of the Weinstein index shifting relations. The Theorem 4.3 states that if $f(x, \xi)$ is a solution of (6.2) with $a$ replaced by $a+2$, then $g(x, \xi)=f(x, \xi)+(2 /(a+1)) \xi f_{\xi}(x, t)$ is a solution of (6.2). Returning to the variable $t$, this becomes (in the above notation)

$$
U^{(a)}(x, t)=\left(1+(t /(a+1)) D_{t}\right) U^{(a+2)}(x, t) .
$$

When this is coupled with the above formula, it is easy to derive $U^{(\beta+2)}(x, t)$ $=t^{-1}(\partial / \partial t)\left\{U^{(\beta)}(x, t)\right\}$, the other Weinstein formula. 
If $a \geqq 1$, the results in [6] are applicable. Now assume that $a=-1-2 m+2 \gamma$ with $m=0,1,2, \ldots$ and $0<\gamma<1$. Then Theorem 4.2 shows directly that a solution of (6.1) is given by

$$
\begin{aligned}
U^{(a)}(x, t)= & \Gamma(-m+\gamma) \sum_{n=0}^{m-1} \frac{\left(\frac{1}{4} t^{2}\right)^{n} P^{n}(x, D) \phi(x)}{n ! \Gamma(n-m+\gamma)} \\
& +\frac{1}{(m-1) !} \frac{\Gamma(-m+\gamma)}{\Gamma(\gamma)}\left(\frac{t^{2}}{4}\right)^{m} P^{m}(x, D) \int_{0}^{1}(1-\sigma)^{m-1} U^{(\gamma)}(x, t \sigma) d \sigma .
\end{aligned}
$$

Sufficient differentiability requirements on $\phi(x)$ are assumed for the validity of this. If $\gamma<1$, we can obtain $U^{(\gamma)}$ from $U^{(\gamma+2)}$ by (6.3).

Finally suppose that $a=-2 m-1, m=0,1,2, \ldots$ The first of the above Weinstein shift relations provides a method for obtaining a solution of the equation in (6.1). That solution cannot satisfy a given initial condition. We now show how the theory of ordinary differential equations can be profitably used here. The Weinstein criterion requiring polyharmonic data if $P=\Delta_{n}$, the Laplacian, falls out.

The ordinary differential equation associated with (6.1) is $Y_{t t}-((2 m+1) / t) Y_{t}$ $=\alpha y$. Its indicial equation has roots 0 and $2 m+2$ which differ by an integer. The theory of ordinary differential equations suggests that (6.1) has a solution operator of the form $O(t, P)=A(t, P)+B(t, P) \ln t$. Substituting into (6.1), $A$ and $B$ must satisfy the operator relations:

$$
\left[B_{t t}-\frac{2 m+1}{t} B_{t}-P B\right] \cdot \phi(x)=0
$$

$$
\left[A_{t t}-\frac{2 m+1}{t} A_{t}-P A-\left(\frac{2 m+2}{t^{2}}\right) B+\frac{2}{t} B_{t}\right] \phi(x)=0 .
$$

If we select $B(t, P)=t^{2 m+2} C(P)$, then (6.4a) holds if $\{P C(P)\} \cdot \phi(x)=0$. The operator $C(P)$ is determined in such a way that $(6.4 \mathrm{~b})$ becomes solvable. From the usual series approach, we can solve for $A$ in powers of $t^{2} P$ if we select

$$
C(P)=\frac{(-1)^{m} P^{m+1}}{2^{2 m+1} m !(m+1) !}
$$

Then (6.4a) holds if we require $\phi(x)$ to satisfy

$$
P^{m+2}(x, D) \phi(x)=0 .
$$

This contains the polyharmonic requirement on the data if $P(x, D)=\Delta_{n}$. A solution operator for the problem can be expressed as

$$
O(t, P)=\sum_{j=0}^{m} \frac{(-1)^{j}(m-j) !}{2^{2 j} j ! m !} t^{2 j} P^{j}+\frac{(-1)^{m} P^{m+1} t^{2 m+2} \ln t}{2^{2 m+1} m !(m+1) !} .
$$

Observe that $\lim _{t \rightarrow 0+} t^{2 m+2} \ln t P^{m+1} \phi(x)=0$ for all $\phi$ satisfying condition (6.5).

\section{REFERENCES}

1. L. R. Bragg, The radial heat polynomials and related functions, Trans. Amer. Math. Soc. 119 (1965), 270-290.

2. - The radial heat equation and Laplace transforms, SIAM J. Appl. Math. 14 (1966), 986-993. 
3. L. R. Bragg, Fundamental solutions and properties of solutions of the initial value radial Euler-Poisson-Darboux problem. J. Math. Mech. 18 (1969), 607-616.

4. L. R. Bragg and J. W. Dettman, Related problems in partial differential equations, Bull. Amer. Math. Soc. 74 (1968), 375-378.

5. - Related partial differential equations and their applications, SIAM J. Appl. Math. 16 (1968), 459-467.

6. - An operator calculus for related partial differential equations, J. Math. Anal. Appl. 22 (1968), 261-271.

7. - Expansions of solutions of certain hyperbolic and elliptic problems in terms of Jacobi polynomials, Duke Math. J. 36 (1969), 129-144.

8. A. Friedman, Parabolic partial differential equations, Prentice-Hall, Englewood Cliffs, N. J., 1964.

9. S. G. Mikhlin, Editor, Linear equations of mathematical physics, Holt, Rinehart and Winston, New York, 1967.

10. E. Rainville, Special functions, Macmillan, New York, 1960.

11. A. Weinstein, Sur le problème de Cauchy pour l'équation de Poisson et l'équation des ondes, C. R. Acad. Sci. Paris 237 (1952), 2584-2585.

12. - "Singular partial differential equations and their applications" in Fluid dynamics and applied mathematics, Gordon \& Breach, New York, 1962.

13. J. B. Diaz, On Cauchy's problem and fundamental solutions, Ann. of Math. Studies, No. 33, Princeton Univ. Press, Princeton, N. J., 1954.

OAKLAND UNIVERSITY, ROCHESTER, MICHIGAN 